\title{
LONG-TERM ASSESSMENT OF THE SALIVARY OXIDATIVE STRESS STATUS DURING ORTHODONTIC TREATMENT WITH FIXED APPLIANCES
}

\author{
Marwa Sameh Shamaa* and Mostafa Mohamed Mansour**
}

\begin{abstract}
Introduction: Saliva has been considered a valuable diagnostic tool for many disorders and diseases.

Objective: The aim of this study was to assess some important biomarkers of oxidative stress in the saliva of patients undergoing fixed orthodontic treatment.

Methods: A total of thirty three subjects (18 females and 15 males) in the age group of 1518 years were enrolled in the study. The salivary samples were collected before fixed appliance placement, 6 months and 1 year after orthodontic treatment onset. Samples were investigated for measurement of malondialdehyde (MDA), nitric oxide (NO) and 8-hydroxydeoxyguanosine (8OHdG) levels.
\end{abstract}

Results: The salivary MDA, NO and 8-OHdG concentrations in the individuals treated with fixed orthodontic appliances did not show significant changes neither after 6 nor after 12 months of treatment compared to the basal values before treatment .

Conclusion: Fixed orthodontic therapy for one year did not induce oxidative damage in the oral cavity.

KEY WORDS: Fixed orthodontic treatment; saliva; oxidative stress; reactive oxygen species.

\section{INTRODUCTION}

Orthodontic treatment of different anomalies often supposes the application of mechanical forces which can trigger a localized inflammation around the teeth subjected to movement. The inflammatory process formation at this level will result in increased free radicals synthesis with consequent oxidative stress. ${ }^{1}$

Oxidative stress reflects an imbalance between the production of reactive oxygen species (ROS) and the capability of the body to cancel out their harmful effects by antioxidants. ${ }^{2}$ The produced ROS provoke

\footnotetext{
* Lecturer of Orthodontics, Faculty of Dentistry, Mansoura University.

** Lecturer of Clinical Pathology, Faculty of Medicine, Mansoura University.
} 
the production of cytokines which will activate the inflammatory cells and play a substantial role in the oxidative damage of the tissues. The unfavourable outcome of excessive ROS and free radicals generation and defects in antioxidant mechanisms and antioxidants in saliva may have a significant role in the appearance and progress of dental caries and many other pathogenic diseases in the oral cavity. ${ }^{3}$ The oxidative stress can be estimated through a series of its biomarkers e.g. malondialdehyde, nitric oxide and 8-hydroxydeoxyguanosine.

Malondialdehyde (MDA) represents a primary biomarker of lipid peroxidation and it is commonly utilized in determining the oxidative damage of lipids-resulting free radicals. ${ }^{4}$ Some earlier investigations reported that the salivary malondialdehyde level was significantly elevated in periodontal diseases and suggested that this elevation has significant contribution to periodontal tissue destruction. ${ }^{4}$

Nitric oxide is a free radical gas and detrimental oxide that presents in the body in small well controlled concentrations. ${ }^{5} \mathrm{NO}$ exists in the salivary glands and gingival crevicular fluid (GCF). Salivary NO has a crucial role in the natural defensive mechanism of the oral cavity against pathogenic bacteria. Massive amount of nitric oxide is related to tissue damage in periodontitis and high NO level indicates more severe inflammation. ${ }^{6}$

8 -hydroxydeoxyguanosine $(8-\mathrm{OHdG})$ is currently used for monitoring the oxidative damage. The level of 8-OHdG has been utilized to evaluate the DNA impairment. It is feasible to estimate cytotoxicity through oxidative stress by measuring the concentration of 8-hydroxydeoxyguanosine. It draws in consideration as a widespread employed molecule in the assessment of oxidative damage that may happen particularly in the periodontal tissues during periodontal inflammation. ${ }^{7}$ Thus, the level of salivary 8 -OHdG seemed to reflect the periodontal health status. ${ }^{8}$ Previous studies were focused on measuring the oxidative stress after short periods of orthodontic appliance placement. ${ }^{1,4}$ So, the aim of this study was to investigate the long term influence of fixed orthodontic mechanotherapy on the salivary oxidative stress biomarkers after 6 months and 1 year of treatment and compare their levels with the pre-treatment values.

\section{MATERIALS AND METHODS}

This prospective clinical study was done to estimate the concentrations of some important salivary oxidative stress biomarkers: MDA, NO and 8-OHdG before, six months and twelve months after fixed orthodontic treatment. The study included thirty three patients (18 females and 15 males), with age ranging from 15 to 18 years old who were recruited from the Department of Orthodontics, Faculty of Dentistry, Mansoura University. The sample size of the thirty three patients was selected based on the results of Youness et $\mathrm{al}^{9}$ and level of absolute precision of $2 \%$ at alpha error of $5 \%$ and study power of $90 \%$. The study was commenced after receiving Informed consents from all the study participants keeping all their rights. The research project was revised and approved by the Ethical Committee, Faculty of Dentistry, Mansoura University (No. 06130818).

The inclusion criteria were as follows: (1) patients who required orthodontic treatment with maxillary first premolar extraction and canine retraction, (2) no systemic or periodontal disease, (3) no previous orthodontic treatment, (4) good oral hygiene, (5) good patient cooperation. All patients were treated with a straight wire technique using metal brackets (3M, Unitek Gemini) that were bonded to the teeth with Transbond XT light cure adhesive (3M Unitek). Oral hygiene instructions were given to the patients before and throughout the study period. Eating or drinking (except water) or chewing gum was avoided at least two hrs prior to saliva collection which was done in the morning 
between 8 and 10 am through spitting into sterile pre-labelled containers. Patients were instructed not to consume any antibiotics or anti-inflammatory drugs at least one week before the collection period. They were also advised not to use dentifrices on the day of collection.

According to the designed protocol, the saliva samples were obtained at different time intervals: before fixed appliance placement and after $6 \&$ 12 months of orthodontic treatment starting. The collected samples were stored at $-20^{\circ} \mathrm{C}$ until the biochemical estimation.

Calorimetric assay kits (Biodiagnostic, Diagnosis and Research Readings) were used for estimation of salivary MDA, NO and 8-OHdG by utilizing spectrophotometric method according to the package inserts.

The statistical analysis of the data was performed using excel (Microsoft office 2013) program and SPSS (Statistical Package For Social Science) program (SPSS, Inc, Chicago, IL) version 20. Shapiro Wilk test was performed to assess the normality of the data. Qualitative data were presented as frequency and percentage. Quantitative data were presented as median, range, mean and standard deviation. For comparison within the same group between three assessment periods; Friedman test was used (for non-parametric data). Spearman rank correlation was used to examine the correlation between different parameters. $\mathrm{P}$ value was considered significant if $\leq 0.05$ at confidence interval $95 \%$.

\section{RESULTS}

The study was carried on 33 patients, including 18 females and 15 males with a median age of 16.5 years (range from 15 to 18 years old) (Table 1).

Table (1): Demographic data of the studied cases.

\begin{tabular}{|c|c|c|c|}
\hline \multicolumn{3}{|c|}{ Data } & $\begin{array}{c}\text { Cases } \\
(n=33)\end{array}$ \\
\hline \multirow{3}{*}{ Gender } & \multirow{2}{*}{ Male } & Number & 15 \\
\cline { 3 - 5 } & \multirow{2}{*}{ Female } & $\%$ & $45.5 \%$ \\
\cline { 3 - 5 } & \multirow{2}{*}{ Age } & $\%$ & 18 \\
\hline \multirow{2}{*}{} & & Mumber & $54.5 \%$ \\
\cline { 3 - 5 } & & SD & $16.5(15-18)$ \\
\hline
\end{tabular}

The levels of salivary MDA, NO and $8-\mathrm{OHdG}$ in patients before, after 6 months and one year of treatment by fixed orthodontic appliances are shown in table (2). The results of this study revealed no significant difference regarding the levels of these biomarkers at the three time intervals.

TABLE (2): Comparison between salivary levels of MDA, NO and 8-OHdG of the studied cases before, after 6 and 12 months of treatment.

\begin{tabular}{|c|c|c|c|c|c|c|c|c|c|c|}
\hline \multirow{3}{*}{ Parameter } & \multicolumn{3}{|c|}{ Before } & \multicolumn{3}{|c|}{ After 6 months } & \multicolumn{3}{|c|}{ After 12 months } & \multirow{3}{*}{$\mathrm{P}$} \\
\hline & \multirow{2}{*}{ Median } & \multicolumn{2}{|c|}{ Range } & \multirow{2}{*}{ Median } & \multicolumn{2}{|c|}{ Range } & \multirow{2}{*}{ Median } & \multicolumn{2}{|c|}{ Range } & \\
\hline & & Min & Max & & Min & $\operatorname{Max}$ & & Min & $\operatorname{Max}$ & \\
\hline MDA & 16.10 & 6.00 & 32.0 & 15.10 & 6.0 & 31.3 & 15.10 & 6.5 & 31.0 & 0.141 \\
\hline NO & 7.90 & 0.12 & 536 & 7.80 & 0.11 & 521.40 & 7.60 & 0.10 & 532 & 0.809 \\
\hline 8-OHdG & 4.93 & 0.07 & 17.00 & 4.91 & 0.09 & 17.0 & 4.90 & 0.06 & 17.3 & 0.249 \\
\hline
\end{tabular}


Correlation between different parameters in cases before treatment is shown in table (3). There was a significant positive correlation between salivary $\mathrm{NO}$ and 8-OHdG levels, while no significant correlations were detected neither between NO and MDA, nor between 8-OHdG and MDA levels.

TABLE (3): Correlation between different studied parameters in patients before treatment.

\begin{tabular}{|l|c|c|c|c|}
\hline \multicolumn{2}{|l|}{ Parameter } & $\begin{array}{c}\text { MDA_ } \\
\text { Before }\end{array}$ & NO_Before & $\begin{array}{c}\text { 8-OHdH_ } \\
\text { Before }\end{array}$ \\
\hline \multirow{2}{*}{$\begin{array}{l}\text { MDA } \\
\text { Before }\end{array}$} & $\mathbf{R}$ & 1.000 & 0.092 & -0.221 \\
\cline { 2 - 5 } & $\mathbf{P}$ &. & 0.610 & 0.217 \\
\hline \multirow{2}{*}{ NO Before } & $\mathbf{R}$ & .092 & 1.000 & $0.495^{* *}$ \\
\cline { 2 - 5 } & $\mathbf{P}$ & .610 &. & 0.003 \\
\hline \multirow{2}{*}{ 8-OHdG } & $\mathbf{R}$ & -.221 & $.495^{* *}$ & 1.000 \\
\cline { 2 - 5 } Before & $\mathbf{P}$ & .217 & .003 &. \\
\hline
\end{tabular}

r correlation coefficient

** significant

Correlation between the studied parameters in patients after 6 months of treatment is shown in table (4). There was a significant positive correlation between salivary NO and $8-\mathrm{OHdG}$ levels but no significant correlations were found neither between $\mathrm{NO}$ and MDA, nor between 8-OHdG and MDA levels.

TABLE (4): Correlation between different parameters in cases after 6 months of treatment.

\begin{tabular}{|l|l|c|c|c|}
\hline \multicolumn{2}{|l|}{ Parameter } & MDA & NO & OHDH \\
\hline \multirow{2}{*}{ MDA } & $\mathbf{R}$ & 1.000 & -.027 & -.260 \\
\cline { 2 - 5 } & $\mathbf{P}$ &. & .880 & .144 \\
\hline \multirow{2}{*}{ NO } & $\mathbf{R}$ & -.027 & 1.000 & $.504^{* *}$ \\
\cline { 2 - 5 } & $\mathbf{P}$ & .880 &. & .003 \\
\hline \multirow{2}{*}{ 8-OHdG } & $\mathbf{R}$ & -.260 & $.504^{* *}$ & 1.000 \\
\cline { 2 - 5 } & $\mathbf{P}$ & .144 & .003 &. \\
\hline
\end{tabular}

r correlation coefficient

** significant
Correlation between the studied parameters in patients after one year of treatment is shown in table (5). There was a significant positive correlation between salivary $\mathrm{NO}$ and $8-\mathrm{OHdG}$ levels but no significant correlations were detected neither between NO and MDA, nor between $8-\mathrm{OHdG}$ and MDA levels.

TABLE (5): Correlation between different parameters in cases after 12 months of treatment.

\begin{tabular}{|l|c|c|c|c|}
\hline \multicolumn{2}{|l|}{ Parameter } & MDA & NO & 8-OHdH \\
\hline \multirow{2}{*}{ MDA } & $\mathbf{R}$ & 1.000 & .094 & -.209 \\
\cline { 2 - 5 } & $\mathbf{P}$ &. & .604 & .243 \\
\hline \multirow{2}{*}{ NO } & $\mathbf{R}$ & .094 & 1.000 & $.501^{* *}$ \\
\cline { 2 - 5 } & $\mathbf{P}$ & .604 &. & .003 \\
\hline \multirow{2}{*}{$8-$-OHdG } & $\mathbf{R}$ & -.209 & $.501^{* *}$ & 1.000 \\
\cline { 2 - 5 } & $\mathbf{P}$ & .243 & .003 &. \\
\hline
\end{tabular}

r correlation coefficient

** significant

\section{DISCUSSION}

Orthodontic treatment produces changes in the periodontal tissues that permit tooth movement. The orthodontic tooth movement relies upon remodelling of periodontal ligament and alveolar bone i.e bone resorption occurs in the compression areas and bone formation takes place in the tension areas. Acute inflammation of the periodontal tissues is among the changes that occur during orthodontic tooth movement. ${ }^{6}$

Saliva is a main vehicle of defense in the oral cavity as it has a fundamental role in protecting \& sustaining the health of the oral tissues. ${ }^{10}$ Free radicals are produced from all the body tissues, including the oral cavity, during the physiological processes. The antioxidant mechanism counterbalances the harmful effect of 
free radicals. The oxidative balance is deteriorated when an alteration in this balance occurs in favour of free radicals resulting in oxidative tissue damage associated with oxidative stress. ${ }^{4}$ Several approaches illustrate the participation of oxidative stress in the pathophysiologic mechanisms of diseases. ${ }^{11} \mathrm{Up}$ to our knowledge, no data are available regarding the oxidative harm that might be generated by long term fixed orthodontic therapy.

The reactive free radicals mostly affect the cellular lipid component. They can cause cell damage through inception of lipid peroxidation resulting in significant change in the cell membrane function and the structural integrity. ${ }^{4}$ Malondialdehyde (MDA) is the chief resultant of lipid peroxidation. Increased malondialdehyde level has been detected in oral leukoplakia, cancer and periodontitis. ${ }^{12}$ Olteanu et $\mathrm{al}^{1}$ reported that the salivary malondialdehyde reached its maximum level at twenty four hours from the orthodontic treatment beginning, while at seven days from the onset of treatment, the MDA concentration was close to the initial value. Oscan et $\mathrm{al}^{4}$ revealed that orthodontic tooth movement did not cause significant difference in the salivary level of malondialdehyde estimated in various treatment periods up to six months indicating no oxidative harm on the lipid components in that timeframe. In the current study, the utilization of fixed orthodontic appliances did not change the level of salivary MDA in patients undergoing treatment for up to one year which may reflect absence of pathological changes in the oral cavity.

The normal cellular metabolism includes the production of free radicals mainly superoxide, hydroxyl and NO. ${ }^{11}$ Exaggerated amounts of salivary nitric oxide may assist in tissue damage in periodontitis. This might be attributed to the influence of NO on osteoclastogenesis. ${ }^{6}$ There is controversy between different studies regarding the level of salivary NO during orthodontic treatment. Alarcon et $\mathrm{al}^{13}$ found that the level of salivary NO increased from the seventh to the thirtieth day after the initiation of fixed orthodontic treatment in adult females and they suggested that future investigations are required to decide if the salivary nitric oxide level could be utilized as a marker for orthodontic tooth movement. Oscan et $\mathrm{al}^{4}$ observed that the salivary nitric oxide level, which reflects the level of periodontal free radicals, was not changed at the $1^{\text {st }}$ month and $6^{\text {th }}$ months of orthodontic treatment. In our study, the salivary NO level displayed no significant change from normal limits after 6 months and one year of orthodontic tooth movement and the level of the periodontal free radicals persisted within the physiological limits for that period of time.

One of the most valuable oxidative stress biomarkers is 8 -hydroxydeoxyguanosine ( $8-\mathrm{OHdG}$ ). It is produced from oxidation of guanine from the damaged DNA. ${ }^{14}$ 8-hydroxydeoxyguanosine represents a marker of oxidative tissue damage in many chronic inflammatory disorders. Detection of 8-OHdG could be used to prove cytotoxicity through oxidative stress. Thus, it has often been utilized as a marker for oxidative stress. ${ }^{15}$ Anusuya et al ${ }^{16}$ confirmed that the salivary $8-\mathrm{OHdG}$ measurement is important to investigate the oxidative damage in patients with periodontitis. They also concluded that the level of 8-hydroxydeoxyguanosine in saliva reflects the oxidative stress, which might reflect the periodontal health state of these individuals. Paredes-Sánchez et $\mathrm{al}^{17}$ found that the concentration of 8-hydroxydeoxyguanosine in saliva of periodontal patients was nearly double that in healthy subjects and its level decreased after treatment of these patients with anti-inflammatory drugs. Ozcan et $\mathrm{al}^{4}$ estimated the level of $8-\mathrm{OHdG}$ in saliva after 3 and 6 months of fixed orthodontic treatment. They observed no significant change during the study period. They declared that the absence of a significant increase in the level of $8-\mathrm{OHdG}$ above the physiological values did not potentiate oxidative stress in both GCF \& saliva and that there is lack of periodontal damage for that period of time. Guler 
et $\mathrm{al}^{18}$ concluded that the level of $8-\mathrm{OHdG}$ in saliva did not increase after one month and three months duration after placement of fixed appliances. In the current work, no statistically significant difference was observed in the salivary 8 - OHdG level between before, 6 months and 1 year after receiving fixed orthodontic treatment.

Conflicting results have been reported concerning the correlation analysis between the different salivary biomarkers. In our work, no significant correlation was detected between neither salivary NO nor 8-OHDG level with the MDA level, at base line, after 6 and 12 months of treatment, while a significant positive correlation was detected between salivary NO and 8- OHdG levels. However, Çanakçi et al ${ }^{19}$ found significant positive correlations between the levels of salivary MDA and $8-\mathrm{OHdG}$ in patients with periodontitis. They suggested that this may be due to that both MDA and 8-OHdG may cause lipid peroxidation damage on DNA and this reflects the increased activity of ROS during periodontal inflammation.

In our study, the absence of significant change in the biochemical markers of oxidative stress: NO, MDA and $8-\mathrm{OHdG}$ in saliva of patients receiving fixed orthodontic therapy at 6 months and one year measurement period as compared to the pretreatment values indicated that orthodontic therapy did not generate induce oxidative damage in the oral cavity for a full year of for a full year of treatment.

\section{CONCLUSION}

Long term assessment of treatment with fixed orthodontic appliances for up to one year, did not reveal considerable changes in the salivary oxidative status and this may reflect the absence of remarkable periodontal inflammation in these patients. Hence, it could be assumed that increased caries risk in orthodontic patients may be attributed to accumulation of food debris around the fixed attachments and not due to oxidative stress.

\section{REFERENCES}

1- Olteanu C, Muresan A, Daicoviciu D, Tarmure V, Olteanu I, Keularts IM. Variarions of some saliva markers of the oxidative stress in patients with orthodontic appliances. Fiziologia-Physiology. 2009 Sep 1; 19 (3).

2- Peluso I, Raguzzini A. Salivary and urinary total antioxidant capacity as biomarkers of oxidative stress in humans. Pathology research international. 2016 Feb.

3- Dodwad R, Betigeri AV, Preeti BP. Estimation of total antioxidant capacity levels in saliva of caries-free and caries-active children. Contemporary clinical dentistry. 2011 Jan; 2 (1): 17.

4- Atuğ Özcan SS, Ceylan İ, Özcan E, Kurt N, Dağsuyu İM, Çanakçi CF. Evaluation of oxidative stress biomarkers in patients with fixed orthodontic appliances. Disease markers. 2014 Apr.

5- Mobarak EH, Abdallah DM. Saliva nitric oxide levels in relation to caries experience and oral hygiene. Journal of Advanced Research. 2011 Oct 1; 2 (4):357-62.

6- Yamamoto Z, Jaafar IM, Rohaya MA, Abidin IZ, Senafi S, Ariffin ZZ, Ariffin SH. Orthodontic treatment effects on inflammatory marker profiles in saliva before and after 2 archwire changes. In AIP Conference Proceedings 2013 Nov 27, 1571(1): 208-14.

7- Shigenaga MK, Gimeno CJ, Ames BN. Urinary 8-hydroxy-2'-deoxyguanosine as a biological marker of in vivo oxidative DNA damage. Proceedings of the National Academy of Sciences. 1989 Dec 1; 86 (24):9697-701.

8- Takane M, Sugano N, Iwasaki H, Iwano Y, Shimizu N, Ito $\mathrm{K}$. New biomarker evidence of oxidative DNA damage in whole saliva from clinically healthy and periodontally diseased individuals. Journal of periodontology. 2002 May; 73 (5):551-4.

9- Youness SR, Kaddah MA, Rahman TA. Total Salivary Antioxidant Capacity in a group of Egyptian Children under Active Orthodontic Treatment. IOSR Journal of Dental and Medical Sciences. 2015; 14:105-10.

10- Dhiman S, Gaur A, Maheshwari S, Khan S. The relevance of physico-chemical and diagnostic properties of saliva during orthodontic treatment. International Journal of Contemporary Dental and Medical Reviews. 2014; 1:5.

11- Akalın FA, Baltacıŏglu E, Alver A, Karabulut E. Lipid peroxidation levels and total oxidant status in serum, saliva and gingival crevicular fluid in patients with chronic 
periodontitis. Journal of clinical periodontology. 2007 Jul; 34(7):558-65.

12- Rai B, Kharb S, Jain R, Anand SC. Salivary lipid peroxidation product malonaldehyde in various dental diseases. World Journal of Medical Sciences. 2006; 1 (2):100-1.

13- Alarcon JA, Martin C, Nuez A and Gonez G. Nitric oxide levels in saliva during early orthodontic tooth movement. IADR conference general session.

14- Buczko P, Zalewska A, Szarmach I. Saliva and oxidative stress in oral cavity and in some systemic disorders. Journal of physiology and pharmacology. 2015.66 (01): 3-9.

15- Shigenaga MK, Gimeno CJ, Ames BN. Urinary 8-hydroxy-2'-deoxyguanosine as a biological marker of in vivo oxidative DNA damage. Proceedings of the National Academy of Sciences. 1989 Dec 1; 86 (24):9697-701.

16- Anusuya S, Prabhuji M, Lazarus F, Shaeesta and Babrawala I. Estimation of 8-Hydroxy-deoxyguanosine
(8- OHdG) in Saliva as a Marker of Oxidative Stress in Patients with Chronic Periodontitis: Preliminary Data. Journal of the International Academy of Periodontology. 2017.19 (3): 95-100.

17- Paredes-Sánchez E, Iranzo-Cortés JE, Almerich-Torres T, Bellot-Arcís C, Almerich-Silla JM. Meta-Analysis of the Use of 8-OHdG in Saliva as a Marker of Periodontal Disease. Disease markers. 2018.

18- Guler C, Toy E, Ozturk F, Gunes D, Karabulut AB, Otlu O. Evaluation of salivary total oxidant-antioxidant status and DNA damage of children undergoing fixed orthodontic therapy. The Angle Orthodontist. 2014 Jun 20; 85(2): 239-44.

19- Çanakçi CF, Çanakçi V, Tatar A, Eltas A, Sezer U, Çiçek Y, Oztas S. Increased salivary level of 8-hydroxydeoxyguanosine is a marker of premature oxidative mitochondrial DNA damage in gingival tissue of patients with periodontitis. Archivum immunologiae et therapiae experimentalis. 2009 Jun 1; 57(3):205-11. 